\title{
Quarkyonic matter from hydro and rapid freeze out
}

\author{
L. P. Csernai ${ }^{* 1,2,3}$, Yun Cheng ${ }^{1,2}$, S. Horvát ${ }^{2}$, V. K. Magas ${ }^{2,4}$, I. N. Mishustin ${ }^{1,5}$, \\ E. Molnár ${ }^{1}$, D. Strottman ${ }^{1,6}, \mathbf{M}$. Zétényi $\mathbf{i}^{2,3}$ \\ Affiliation \\ ${ }^{1}$ Frankfurt Institute for Advanced Studies, Johann Wolfgang Goethe University \\ Ruth-Moufang-Str. 1, 60438 Frankfurt am Main, Germany \\ ${ }^{2}$ Institute for Physics and Technology, University of Bergen, 5007 Bergen, Norway \\ ${ }^{3}$ MTA-KFKI, Research Inst. of Particle and Nuclear Physics, 1525 Budapest, Hungary \\ ${ }^{4}$ Departament d'Estructura i Constituents de la Materia, Universitat de Barcelona, \\ Diagonal 647, E-08028 Barcelona, Spain \\ ${ }^{5}$ The Kurchatov Institute, Acad. Kurchatov Sq. 1, Moscow 123182, Russia \\ ${ }^{6}$ Theoretical Division, Los Alamos National Laboratory, Los Alamos, New Mexico 87545, USA \\ E-mail: csernaidfias.uni-frankfurt.de
}

\begin{abstract}
Quarkyonic matter is a predicted phase between deconfined ideal QGP and Hadronic matter where the dominant degrees of freedom are quarks. Collective flow measurements indicate that the flow developed in QGP, as flow measurements scale with the constituent quark numbers. The possible reasons for the observed constituent quark number scaling were analyzed, arriving to the conclusion that collective flow must have frozen out early when quarks were the dominant constituents of matter.
\end{abstract}

5th International Workshop on Critical Point and Onset of Deconfinement - CPOD 2009,

June 08 - 122009

Brookhaven National Laboratory, Long Island, New York, USA

\footnotetext{
*Speaker.
} 


\section{Introduction}

At low energy heavy ion collisions nucleons and light composites like $d$ and $\alpha$, reach thermal, chemical and then flow equilibrium before Freeze Out (FO). The same flow velocity and same temperature is reached for all hadronic fragments. Due to different masses of $p, d$, and $\alpha$, the thermal energy for the these fragments are the same but their random thermal velocities are large for $p$-s and smaller for the more massive particles. Thus, the heavier particles showed stronger collective flow than the lighter ones.

At higher energies the situation is different, although at the first sight the composite hadrons show also stronger collective, "elliptic flow" proportionally to their Constituent Quark (CQ) number versus the transverse momentum per constituent quark.

Quarkyonic matter [1] is a predicted phase between the deconfined ideal QGP, and the Hadronic matter where chiral symmetry is broken. In Quarkyonic matter the dominant degrees of freedom are quarks, confined into small quark clusters or prehadrons while chiral symmetry is still not broken, so these clusters are relatively light and of nearly equal mass. This picture is in surprisingly good agreement with the collective flow measurements, which indicate that the flow developed in a phase with small viscosity and relatively light and nearly equal mass constituents, CQs or pre-hadrons.

The existence of Quarkyonic matter is predicted as a possibly stable phase at high baryon chemical potentials, however it cannot be excluded that even at lower net baryon charges typical at freeze out of high energy heavy ion collisions, such intermediate phase occurs during the simultaneous FO and hadronization.

\section{Modeling hadron flow}

To describe an ultra-relativistic heavy ion collision in the RHIC and LHC energy region we developed a Multi Module Model, with an initial state formed via a Yang-Mills flux tube model, considering all aspects of a finite impact parameter collision realistically in $3+1$ dimensions. Then the subsequent Fluid Dynamical (FD) development assumed a pure ideal QGP described by the MIT Bag Model equation of state (EoS). The description is completed by a rapid FO and Hadronization in a narrow space-time layer of 1-2 fm/c extent. In most calculations (the normal of) this layer is time-like, and starts when the formation of Quarkyonic matter is expected.

The initial state model divides up the transverse plane into a grid $\left\{x_{i}, y_{j}\right\}$, and then describes the initial stages of the collision as a sum independent streak-streak collisions corresponding to different transverse coordinates. Such a describtion leads to the development of a skewed initial state, generating both elliptic flow component, $v_{2}$, and 3rd flow component, seen as a flow in direction perpendicular to the standard directed flow in $v_{1}$ flow component [2]. The details of the initial state were recently adjusted to higher resolution Computational FD (CFD) calculations, treating the description of surface profiles more realistically. This initial state has both an excentricity and tilt, verified experimentally also [3].

The CFD model uses the PIC method with random placing of the marker particles to minimize the development of grid connected numerical "ringing" instabilities. 
The numerical viscosity of the model is not negligible. With a cell size of $0.5-1 \mathrm{fm}$ it is of the order of the theoretically predicted viscosity of QGP and it is close to the limiting minimal viscosity conjectured in string model estimates.

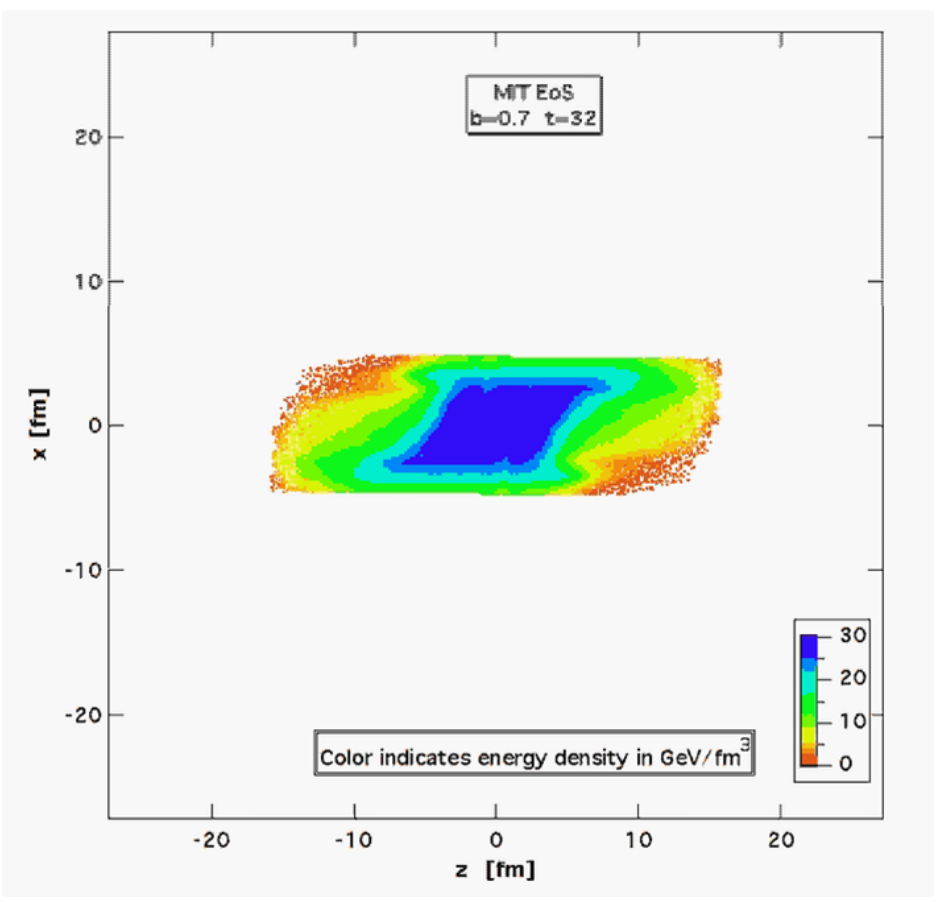

Figure 1: (color online) Initial state of the CFD calculation for a $\mathrm{Au}+\mathrm{Au}$ at energy $65+65 \mathrm{~A} \mathrm{GeV}$, and impact parameter $b=70 \%$ of $b_{\max }$. About 5 million Lagrangian fluid cells are moving in the calculation, using the MIT Bag model EoS.

The initial state for CFD, generated by a Yang-Mills flux tube model [2], is shown in Figure 1. The calculation is concluding at FO at a temperature of $T \approx 200 \mathrm{MeV}$, but the calculation can be continued longer, until pressure is zero for $90 \%$ of the cells. The structure and asymmetries of the initial state are maintained in nearly perfect fluid expansion when the FO is reached.

At this relatively early FO the current quarks have high thermal velocities. In a 1-2 fm/c FO layer, these recombine into pre-hadronic clusters, and deconfinement over the whole QGP volume ceases to exist. The gluons disappear, and their role becomes negligible. A weakening background field may remain among the dominant CQs or pre-hadronic clusters. Thus, we reach the upper boundary (high T) of the Quarkyonic matter.

Collective flow freezes out as the QGP condensates into pre-hadrons, then the matter develops further in the Quarkyonic phase until the chiral symmetry is completely broken and the final hadronic masses are achieved, while the background field disappears.

At the end of the FD stage, when the flow freezes out, the flow velocity (disregarding the random thermal velocities) shows strong signs of the collective flow, as well as fluctuations arising from the structure of the initial state, see Figure 2. 


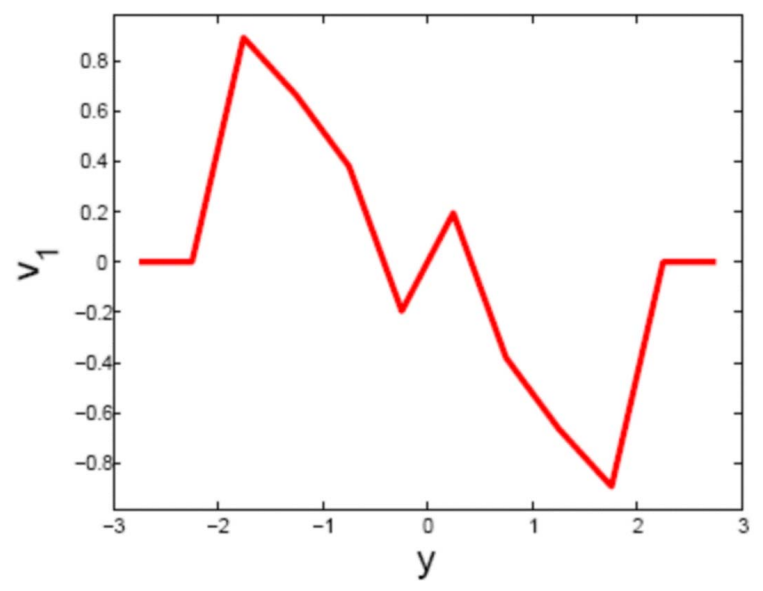

Figure 2: (color online) Harmonic flow parameter, $v_{1}$ for a noncentral collision at finite impact parameter, $b=0.3 b_{\max }$. The random thermal velocities are not taken into account, consequently the flow parameters and their fluctuations are overestimated.

\section{Rapid and simultaneous FO and hadronization}

We consider FO in a narrow layer where the matter is in the Quarkyonic phase. No mixed phase can develop as time is not sufficient for chemical phase equilibration.

Crossing this layer, energy-momentum and baryon charge conservation should be satisfied, as well as entropy should not decrease. From this requirement alone it follows that the CQ masses should be small to fit the experimentally observed hadron multiplicities [4].

This result is also supported by another unrelated observation. Recently we have seen that the observed constituent quark number (NCQ) scaling of elliptic flow can be reproduced in a parton cascade model assuming roughly equal masses and momentum distributions [5] for the participant quarks, which resulted in a constituent quark number scaling of collective flow. So that $v_{n}\left(p_{t}\right)$, for mesons $(n=2)$ and baryons $(n=3)$, becomes

$$
\begin{aligned}
v_{2, M}\left(p_{t}\right) / 2 & =v_{2, q}\left(p_{t} / 2\right) \\
v_{2, B}\left(p_{t}\right) / 3 & =v_{2, q}\left(p_{t} / 3\right) .
\end{aligned}
$$

This result assumes that the mass of CQ quarks does not change. In a local homogeneous source this scaling applies, [5], however, the final observed flow arises from a hypersurface or a FO layer. The space-time features of the source are determining the final flow asymmetries. Comparing model parameters to observations requires that we take into account the random thermal velocities, and average for the range of impact parameters measures, as well as for the freeze out times which may fluctuate event by event,

Elliptic flow, $v_{2} / n_{q}$, from experiments [6], for, $\pi, \mathrm{K}, \mathrm{K}^{*}, \mathrm{p}, \Lambda$ and $\Xi$ shows relatively good scaling versus $p_{t} / n_{q}$ and even more uniform behavior versus $K E_{T} / n_{q}=\left(m_{t}-m\right) / n_{q}$.

The lower momentum part of the spectrum, up to about $p_{t} / n_{q}=0.5 \mathrm{GeV} / \mathrm{c}$, is dominated by the collective elliptic flow, the $v_{2} / n_{q}$ curves versus $p_{t} / n_{q}$ start to rise weaker for heavier hadrons then bend up and become steeper, so that a spread among the different mass particles remains the 


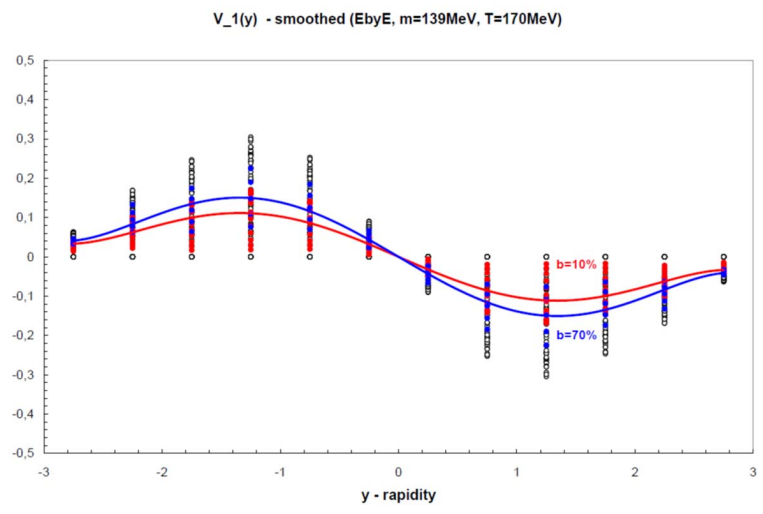

Figure 3: (color online) Harmonic flow parameter, $v_{1}$ for collisions event by event at different impact parameters and different FO times. The random thermal velocities are taken into account, consequently the flow parameters are strongly reduced.

same and the heaviest ones are the lowest among the curves and are significantly bent. At the start the $v_{2} / n_{q}$ curves for different mass hadrons are $\Delta p_{t} / n_{q} \approx 0.2 \mathrm{GeV} / \mathrm{c}$ apart.

The same data plotted versus $\left(m_{t}-m\right) / n_{q}$ start up straight, the different mass hadronic fragments are not separated and not bent.

At higher momenta, around $p_{t} / n_{q}=1 \mathrm{GeV} / \mathrm{c}$ the $v_{2} / n_{q}$ curves of different fragments saturate and reach $v_{2} / n_{q} \approx 0.08$ at $p_{t} / n_{q} \approx 1.8$. Here the error bars are getting larger indicating that smaller multiplicity at high momenta results in weaker collective flow. The NCQ scaling is observed at all impact parameters and source excentricities.

If at the FO stage the matter is made of different hadronic fragments, of different temperatures and flow velocities, already the overall scaling above is difficult to reproduce and only detailed dynamical kinetic recombination models can eventually describe the NCQ flow scaling [5] .

\subsection{Scaling of constituent quarks}

We want to analyze what conclusions we can draw from the NCQ scaling and from its detailed properties. We can assume that flow freezes out in the Quarkyonic matter stage, when CQs have reached the roughly equal (non-zero) masses, $m_{c q}$, and the same 4-velocity distribution $u_{i}(x)=\gamma_{i} v_{i}$ [7]. Then the flow asymmetry does not change any more, but CQ-s recombine into hadrons, with masses, $m_{H}$, and thus becoming more massive (in case of most final primary hadrons). According to the analysis of experiments [8], the hadron chemical Freeze Out (FO) happens when the internal energy of the hadrons $E_{H}$ (including their rest masses and thermal energies) is $E_{H} / N_{H}=1.08 \mathrm{GeV}$ for a final hadron. Based on the observed mesont to baryon ratio at RHIC energies, the final hadron number, $N_{H}$ can be translated to the number of constituent quarks as $N_{c q}=2.25( \pm 0.1) N_{H}$, thus $E_{H} / N_{c q}=0.48 \mathrm{GeV}$ at hadronic FO.

To make a transparent study of the NCQ scaling we utilize a few source model, assuming few thermally equilibrated sources including different mass hadrons, with given flow velocities and temperatures. Following refs. $[9,10]$ and sections 8.3.2-4 of [11] let us consider the FO layer or 
hypersurface as composed of 3 elements in the transverse, [x,y], plane. In ref. [12] a similar few source model was worked out for 4 sources for the study of elliptic flow.

At FO we assume that the sources contain constituent quarks of mass $m \approx 100 \mathrm{MeV}$. Two of these sources, the side sources, labeled by "s", with mass number $A_{s}$, move up and down in the reaction plane (x direction) while the third source, the center source, labeled by "c", with mass number $A_{c}$, is on the y-axis and its flow velocity is assumed to be negligible. In ref. [12], more realistically, two central sources with smaller $+/$ - $y$-directed velocities were considered.

The side sources have collective momenta per nucleon: $\vec{p}_{s}=\left(p_{t}^{s}, 0,0\right)$ and $-\vec{p}_{s}$ where $[x, z]$ is the reaction plane and $z$ is the beam axis. Their flow velocities are

$$
u^{\mu}(s)=\gamma_{x}\left(1, \pm v_{x}, 0,0\right)=\left(\gamma_{x}, \pm u_{x}, 0,0\right)
$$

In the Quarkyonic matter phase [1] there are no gluons and single color charges, but meson or baryon type of quark configurations, which gradually reach the final hadron masses when free hadrons are formed at fixed $E_{H} / N_{H}$. One can assume that kinetic degrees of freedom and flow freeze out at this point together with hadronization or even later. We have, however, seen in section 3.1, that NCQ scaling of flow data does not support this assumption. It rather indicates that the quark constituents have decisive role in the flow development.

Now following ref. [12], let us calculate $v_{2}$ at transverse momentum $p_{t}$. For each particle, emitted towards the azimuth angle $\Phi$, we get $\cos 2 \Phi=\cos ^{2} \Phi-\sin ^{2} \phi=\left(p_{x}^{2}-p_{y}^{2}\right) / p_{t}^{2}$. After averaging this leads to the definition of $v_{2}$ as $v_{2}=\left\langle p_{x}^{2}-p_{y}^{2}\right\rangle /\left\langle p_{x}^{2}+p_{y}^{2}\right\rangle$ The symmetric central source does not contribute to the asymmetry in the nominator, but it does to the symmetric integral in the denominator. Using the expression given in ref. [12] in simplified form (for 3 sources) we obtain

$$
v_{2}\left(y, p_{t}\right)=\frac{I_{2}\left(z_{x}\right)}{I_{0}\left(z_{x}\right)+\frac{A_{c}}{2 A_{s}} \exp \left(E\left(\frac{\gamma_{x}}{T_{s}}-\frac{1}{T_{c}}\right)\right.}
$$

where $z_{x}=p_{t} u_{x} / T_{s}$, and $T_{s}\left(T_{c}\right)$ is the temperature of the side (central) source. We will see the scaling behavior of the transverse flow in the framework of the three source model in the next sections. The energy of an emitted particle is $E=p^{0}=\sqrt{m^{2}+\vec{p}^{2}}=\sqrt{m^{2}+p_{t}^{2}} \cosh \left(y_{p}\right)$, thus in the central transverse plane, where the particle rapidity is $y_{p}=0$, the paticle energy is reduced to $E=m_{t}$, and

$$
v_{2}\left(p_{t}\right)=\frac{I_{2}\left(z_{x}\right)}{I_{0}\left(z_{x}\right)+\frac{A_{c}}{2 A_{s}} \exp \left(\sqrt{m^{2}+p_{t}^{2}}\left(\frac{\gamma_{x}}{T_{s}}-\frac{1}{T_{c}}\right)\right)}
$$

The y-directed flow is neglected in this simplified model, thus the feature that heavier particles at low $p_{t}$ may show negative or decreasing $v_{2}$ [12], is not present.

If $T_{c}<T_{s} /\left(\gamma_{x}-u_{x}\right)$ the asymptotic behavior, $p_{t} \gg m, T_{s}, T_{c}$ of this model gives $v_{2}(z \rightarrow \infty)=1$ for all particle masses This is the same result the two side sources would give without thermal smearing. The function $v_{2}\left(p_{t}\right)$ reaches half saturation at higher $p_{t}$ for higher source temperatures, $\left(T_{s}=T_{c}\right)$, independent of particle masses.

For equal source temperatures $T_{s}=T_{c}$, and composite masses of $m_{H}=n_{q} \times m$ the model results in identical $v_{2}\left(p_{t}\right)$ functions, independent of mass $m$ and exactly the same curve for $n_{q}=1,2,3$.

The transverse momentum at half-saturation, $p_{h s}$, is roughly proportional to the source temperature, $p_{h s} \approx 10 T_{s} / c$, in the $100-250 \mathrm{MeV}$ temperature range. If $T_{c} \approx T_{S} /\left(\gamma_{x}-u_{x}\right)$ the structure of 
the $v_{2}\left(p_{t}\right)$ function may have a local maximum and a local minimum before it tends asymptotically to 1 .

If $T_{c}>T_{s} /\left(\gamma_{x}-u_{x}\right)$ the $v_{2}$-flow starts increasing up to a local maximum then decreases and asymptotically tends to $v_{2}(z \rightarrow \infty)=0$. The hotter thermal source overwhelms the cooler side sources at asymptotically high momenta. The position of the maxima is weakly dependent of the particle mass, but the value of $v_{2}$ is mass dependent, it is higher for smaller masses.

Let us now consider from the same sources (same $u_{x}$ ) a hadron $(\mathrm{H})$ composed of $n_{q}$ constituent quarks, and introduce $p_{t} / n_{q}$ as new variable, i.e. $p_{t n} \equiv p_{t} / n_{q}$ or $p_{t} \equiv n_{q} p_{t n}$. The particle mass is $m_{H}$, then for the composite particles

$$
\frac{v_{2}\left(p_{t n}\right)}{n_{q}}=\frac{1}{n_{q}} \frac{I_{2}\left(z_{x}\right)}{I_{0}\left(z_{x}\right)+\frac{A_{c}}{2 A_{s}} \exp \left(\sqrt{m_{H}^{2}+p_{t}^{2}}\left(\frac{\gamma_{x}}{T_{s}}-\frac{1}{T_{c}}\right)\right)}
$$

where $m_{t}=\sqrt{m_{H}^{2}+p_{t}^{2}}=\sqrt{m_{H}^{2}+\left(n_{q} p_{t n}\right)^{2}}$ is the transverse mass of the composite particle, and $z_{x}=n_{q} p_{t n} u_{x} / T_{s}$, is the argument of the Bessel function, $I_{n}$. Sinilarly to the $v_{2}$ vs. $p_{t}$ curves the $v_{2} / n_{q}$ vs. $p_{t} / n_{q}$ curves start out also with near to zero derivative at the origin.

We can introduce the variable $E_{t n} \equiv\left(m_{t}-m\right) / n_{q}=\left(\sqrt{m_{H}^{2}+p_{t}^{2}}-m_{H}\right) / n_{q}$, used in presenting constituent quark number scaling results. Using $E_{t n}$ the transverse momentum per CQ can be expressed as $p_{t n}=\sqrt{E_{t n}^{2}+2 E_{t n} \frac{m_{H}}{n_{q}}}$. and the argument of the Bessel functions will become $z_{x}=$ $u_{x} n_{q} \sqrt{E_{t n}^{2}+2 E_{t n} \frac{m_{H}}{n_{q}}} / T_{s}$. This way we can study explicitly the NCQ scaling properties of the few source model.

The arising $v_{2} / n_{q}$ vs. $\left(m_{t}-m\right) / n_{q}$ curves start steeply rising immediately at the origin. Thus, this simple model reproduces the observed feature that NCQ scaling versus $\left(m_{t}-m\right) / n_{q}$ is not bent at the beginning as the curve versus $p_{t} / n_{q}$, and the different mass hadrons fall on the same line at very low $p_{t}$. At the same time with increasing transverse momentum the different hadrons do not scale with NCQ. This is, however, the only feature of the NCQ scaling observations, which is reproduced by the thermal, few source model.

The temperature dependent features listed for the few source model above, have fixed saturation points and a temperature and mass dependent structures. These features do not yield NCQ scaling, primarily due to the mass independent saturation points in the model, and also by the fact that the structure of the observables is determined by the mass-temperature ratio and the temperature does not scale with NCQ either.

Two special results illustrate the inability of reproducing the NCQ scaling in the thermal few source model.

\subsection{Flow against thermal motion}

As mentioned above, if $T_{c} \approx T_{s} /\left(\gamma_{x}-u_{x}\right)$ the structure of the $v_{2}\left(p_{t}\right)$ function may have a local maximum and a local minimum before it tends asymptotically to 1 . The function $v_{2}\left(p_{t}\right)$ is not increasing linearly in the $p_{t}=1-2 \mathrm{GeV} / \mathrm{c}$ region, thus the three source model in this balanced situation reproduces qualitatively the experimentally observed saturation of $v_{2}$, as a local maximum in the distribution. The part of the curves at high momenta, $p_{t}>2 \mathrm{GeV} / \mathrm{c}$, are not relevant for the hydrodynamical description of the elliptic flow. Due to the non-linear behavior of $v_{2}\left(p_{t}\right)$ the scaling of elliptic flow with NCQ is not realized in the three source model with balanced parameters. 
The three source model with hadrons coalesced from CQs, assumes that all hadrons are having the same flow velocity, but the sources are in thermal equilibrium, so the thermal energies of different hadrons are equal within each source. Irrespective of taking the same CQ masses and compose the hadrons or taking the final hadron masses, the thermal equilibrium assumption at flow freeze out is not reproducing the observed NCQ scaling. In the balanced flow situation this non scaling is even stronger than in the examples presented in the previous sections.

Presenting the scaling data in terms of $E_{t n}=\left(m_{t}-m\right) / n_{q}$, does not change this basic conclusion, although the low $E_{t n}$ behaviour of the curves agrees qualitatively with the shape of the observed spectra better.

In conclusion, taking into account thermal equilibrium among different mass fragments, with different local source temperatures, cannot reproduce the observed data.

\subsection{Saturation of the flow}

Few source models with same temperature sources and some simplified hydro models have typically a linear $v_{2}$ vs. $p_{t}$ dependence at high $p_{t}$. We have seen that the three source model with colder central source at asymptotically high momenta tends to the saturation value, $v_{2}=1$, irrespective of particle mass if the flow froze out when the different masses were formed.

Smaller local maxima of $v_{2}$ may also appear at finite $p_{t}$ with specially chosen source parameters. Although, these local maxima may be dependent on the particle mass, these do not show the NCQ scaling either.

Another way to achieve saturation of $v_{2}$ at values smaller than one is to include additional sources flowing in the transverse, $y$-direction, e.g. 4 sources [12] or 5 sources. By choosing the source parameters adequately we can get any asymptotic saturation value between zero and one. This saturation value of $v_{2}$ will then be also universal and it will not show the NCQ number scaling.

We can extend this line of argumentation to an increasing number of sources, and arrive at the conclusion that the general tendency of NCQ scaling of flow for different mass hadrons is not supported by the fluid dynamical model, if the different mass hadrons are in thermal and chemical equilibrium with each other when (or before) the flow freezes out.

\section{Development of Fragments After CQ Flow}

We have seen in the previous section that freeze out of flow when thermal and chemical equilibrium has already developed among different mass hadrons, cannot reproduce the observed NCQ number scaling. Transport models can explain the observations only if strong assumptions on the momentum distributions of the CQs are assumed. Another important comment is that in the transport theoretical approximations, which explained the NCQ scaling thermal equilibrium was not considered, rather the properties of the collision integral and molecular chaos were exploited.

The existence of Quarkyonic matter at or before freeze out, would suggest flow and thermal equilibration at lower and approximately equal CQ masses, and given CQ numbers. At this stage there is still a background field, in local equilibrium with the CQs, and the CQs are starting to recombine into the final pre-hadrons. The hadronization and the corresponding mass increase is going to the cost of the energy of the background field and not via kinetic re-thermalization! This 
keeps the flow velocities unchanged during this process, which is also an expression of the QGP being a nearly perfect fluid.

This stage is then followed by mass gain of the pre-hadrons via absorbing all energy of the rest background field. This mechanism also explains the observed uniform feature that hadron chemical Freeze Out happens when the internal energy of the hadrons $E_{H}$, including their rest masses and thermal energies, reaches $E_{H} / N_{H}=1.08 \mathrm{GeV}$ for a final hadron, as mentioned in section 3.1.

The Freeze Out and hadronization takes place in a space-time layer of about 1-2 fm thickness (for time-like surfaces 1-2 fm/c). In fluid dynamical models, most of the time this layer is reduced to a space-time hyper-surface, which, according to the Cooper-Frye formula, is used to calculate the observables.

Following the FO of the flow, the relatively light constituent quarks become heavier due further absorption of the remaining gluon field energy (e.g. represented by the Bag constant B), while recombining to hadrons. We may assume that this absorption process is also proportional to $N_{c q}$ as baryons occupy more volume than mesons. At the same time the thermal energy has to decrease. The total energy is conserved, and as our sources are representing different spatial domains at FO the, energy exchange among different sources is small.

Then flow asymmetry does not change any more during this process, while in a medium, $p_{t}$ is not necessarily conserved. The observed scaling indicates that in this recombination and mass increase the transverse kinetic energy, $K E_{T}=\left(m_{t}-m\right)$ might be conserved, so $\left(m_{t}-m\right) / n_{q}$ is the scaling variable.

Is our picture of recombination to hadrons and mass gain after FO from thermally equilibrated, light CQs reconcilable with the constancy of $\left(m_{t}-m\right) / n_{q}$ ?

For a CQ

$$
\begin{aligned}
E_{t} & \equiv \sqrt{m^{2}+p_{t}^{2}}-m=m\left(\sqrt{1+u_{t}^{2}}-1\right) \\
& \sim m \frac{1}{2} u_{t}^{2}: \text { for } u_{t} \ll 1 \\
& \sim m u_{t}: \quad \text { for } u_{t} \gg 1
\end{aligned}
$$

The scaling flow indicates dependence on transverse energy, not only on the flow velocity, $u_{t}$, but also on the constituent quark mass, $m$.

Thus, if during this process $m$ increases, $u_{t}$ must decrease, However, the final stages of hadronization do not change the flow-asymmetry, but locally the constituent quarks complete their "dress up" in their local region by redistributing energy to reach equilibrium. In other words the background field condenses around quark clusters, without changing the flow distribution. At this change the pressure gradients are already negligible (FO) so the azimuthal distribution of the condensation nuclei (CQs) does not change. Thus all collective flow harmonics remain unchanged.

Although, this picture of recombination to hadrons and mass gain after FO from thermally equilibrated, light CQs, is not a unique possible explanation for the observed NCQ scaling, our model studies in simple fluid dynamical models indicate that other possibilities with thermal and chemical equilibration at later stages, need very special choices of source parameters, and these parameter choices vary for different colliding systems. 
Thus, in fact this is the only physically acceptable choice to explain the data: the observed collective flow freezes out in a Quarkyonic matter stage where constituent quarks are still light and equal in their masses. Mass increase and final hadron formation happen later.

Although, we did not construct here an explicit kinetic model with a disappearing background field to describe this freeze out and hadronization stage after the development of the collective flow, it is reasonable to assume that most models on this basis would reproduce the observed NCQ scaling invariance of flow parameters.

\section{References}

[1] L. McLerran and R. Pisarski, Nucl.Phys. A796, 83 (2007); Y. Hidaka, L. McLerran and R. Pisarski, Nucl.Phys. A808, 117 (2008).

[2] V. Magas, L.P. Csernai and D. Strottman Phys. Rev. C64 (2001) 014901, and Nucl. Phys. A 712 (2002) 167-204

[3] M. Lisa, et al., Phys. Lett. B489 (2000) 287, ibid B496 (2000) 1.

[4] M. Zétényi and L.P. Csernai, in preparation.

[5] D. Molnár, Nucl. Phys. A 774 (2006) 257.

[6] R.A. Lacey, et al., Nucl. Phys. A785 (2007) 122, arXiv: nucl-ex/0608046

[7] CQs still have color, but the energy of the arising gluon fields is concentrated around the CQs. Rest g-fields are considered energetically negligible.[1]

[8] J. Cleymans, et al., Phys. Lett. B615 (2005) 50.

[9] L.P. Csernai, G. Fái, J. Randrup, Phys. Lett. 140B (1984) 149,

[10] A. Bonasera, L.P. Csernai, B. Schürmann, Nucl. Phys. A 476 (1988) 159.

[11] L.P. Csernai: Introduction to Relativistic Heavy Ion Collisions, (Wiley, 1994) and http://home.broadpark.no/ ccsernai/Csernai-textbook.pdf

[12] P. Huovinen, et al., Phys. Lett. B 503 (2001) 58. 